\section{BMJ Open Sport \& Exercise Medicine}

\title{
‘Managing My Patellofemoral Pain': the creation of an education leaflet for patients
}

\author{
Christian J Barton, ${ }^{1,2,3,4}$ Michael S Rathleff ${ }^{5,6,7}$
}

To cite: Barton CJ, Rathleff MS. 'Managing My Patellofemoral Pain': the creation of an education leaflet for patients. BMJ Open Sport Exerc Med 2016;2: e000086. doi:10.1136/ bmjsem-2015-000086

- Prepublication history and additional material is available. To view please visit the journal (http://dx.doi.org/ 10.1136/bmjsem-2015000086).

Accepted 2 January 2016

\section{CrossMark}

For numbered affiliations see end of article.

\section{Correspondence to} Dr Christian J Barton; christian@ completesportscare.com.au

\section{ABSTRACT}

Study design: Qualitative, including consultation with international experts and patients.

Purpose: Develop a brief yet comprehensive evidencebased education leaflet to be used as an adjunct in the management of patellofemoral pain (PFP) through consultation with both experts (clinical academics) and individuals with PFP.

Background: Appropriate patient education is an essential component of effective PFP management. However, there are currently no published educational resources for clinicians and researchers treating individuals with PFP to help translate current evidence into clinical practice.

Methods: A preliminary education leaflet titled 'Managing My Patellofemoral Pain' was created using information from the 'Best Practice Guide to Conservative Management of Patellofemoral Pain' and educational content used in published research. Feedback was sought from 21 experts (clinical academics) for accuracy, adequacy and clarity of the information in the leaflet using a semistructured questionnaire, and a number of suggested modifications were made as a result. Further feedback was sought from 20 patients diagnosed with PFP regarding the clarity and adequacy of information contained in the leaflet, and to determine additional educational resource needs.

Results: The leaflet created is titled 'Managing My Patellofemoral Pain' and the main topics of the leaflet are 'What might cause my knee pain?' and 'Treatment options', which are divided into exercise and additional treatments. Patient feedback was positive, and included a number of considerations for further education resource development.

Conclusions: The 'Managing My Patellofemoral Pain' education leaflet may provide a valuable resource for patients, clinicians and researchers to assist the provision of education and translation of the current evidence.

\section{BACKGROUND}

Patellofemoral pain (PFP) is a prevalent multifactorial knee condition, accounting for $16.5 \%$ of all consultations in sports medicine clinics. ${ }^{1}$ We recently created a 'Best Practice Guide to Conservative Management of
What are the new findings

- The 'Managing My Patellofemoral Pain' education leaflet developed through consultation with international experts is the first published freely available educational resource for patients with patellofemoral pain (PFP).

- The leaflet provides a valuable resource for patients, clinicians and researchers to assist the provision of education and to help translate the current evidence base into clinical practice.

- The leaflet aims to impact on health behaviour, knowledge retention and patient outcomes, and research is now needed to confirm its potential related to these outcomes.

Patellofemoral Pain' based on current level 1 evidence (ie, high-quality systematic reviews) and international expert opinion. ${ }^{2}$ Multimodal interventions possess the strongest evidence in the longer term management of PFP, and commonly consisted of quadriceps and gluteal exercises, stretching, massage, patellar mobilisation and patellar taping. ${ }^{2}$ International expert opinion highlighted that tailoring this multimodal approach, along with appropriate patient education, is essential for effective PFP management. ${ }^{2}$ Importantly, this recommendation fits with previous evidence supporting the efficacy of patient education alongside multimodal intervention in conditions including hip and knee osteoarthritis. ${ }^{3}$

The question is how to provide patient education in clinical practice? Previous research indicates poor knowledge retention when information is only provided verbally, ${ }^{4}$ highlighting the need for education resources to facilitate effective education of PFP patients, and the translation of the current evidence base into clinical practice. Currently, no published educational resources for PFP exist. Previously published studies have used educational interventions as comparisons ${ }^{6}$ or in conjunction with other treatments. ${ }^{7-9}$ However, the material is rarely 
described in enough detail to allow use outside the study, and is based on the researcher's own experience without input from other experts.

The purpose of this study was to develop a brief yet comprehensive evidence-based education leaflet to be used as an adjunct in the management of PFP through consultation with both experts (clinical academics) and individuals with PFP. The hope is that this resource will improve the translation of the current evidence base related to PFP management into clinical practice.

\section{METHODS}

\section{Initial development ( phase 1)}

A preliminary education leaflet was created using information from the 'Best Practice Guide to Conservative Management of Patellofemoral Pain'2 and educational content used in published research. ${ }^{689}$ To ensure that the leaflet was comprehensive, attempts were made to include all information from the 'Best Practice Guide to Conservative Management of Patellofemoral Pain'² in the leaflet, with language simplified to enhance readability. Information related to specific manual therapy options which may assist in the management of PFP was not included, since this was largely based on expert opinion and experts have previously stated that manual therapy specifics and needs are highly variable. ${ }^{2}$

\section{Consultation with international experts (phase 2)}

Using semistructured questions constructed in 'Survey Monkey,' feedback was sought from international experts for accuracy, adequacy and clarity of the information in the leaflet (see online supplementary file 1). Defining expertise is difficult, but for the purpose of this study experts were required to have had at least 5 years clinical experience, be actively involved in PFP research and be contributing to specialist international meetings. It was felt that experts with a good blend of clinical experience and research knowledge would provide the most valuable information regarding potential validity and improvements related to the leaflet. Initial invitations were sent to attendees of the 3rd International Patellofemoral Pain Retreat held in Vancouver, 2013. ${ }^{10}$ Twenty-one experts volunteered, with participants working in 'The USA' $(n=5)$, 'The UK' $(n=4)$, 'The Netherlands' $(n=3)$, 'Canada' $(n=3)$, 'Brazil' $(n=3)$, 'Denmark' $(n=1)$ and 'Australia' $(n=1)$. Academically, four were professors, three were associate professors, three were assistant professors, nine were $\mathrm{PhD}$ candidates, one was a master's student, and one was a clinical scientist. Average research experience ranged from 2 to 20 (mean=7.3) years. Importantly, 14 of the 21 participants were still actively treating patients with PFP, seeing on average 7 new patients with PFP per month. Clinical backgrounds included physiotherapists $(n=11)$, physical therapists $(n=3)$, athletic trainers $(n=2)$, medical doctors $(n=4)$ and one podiatrist.

Survey data were evaluated using a 'Framework Analysis' approach. ${ }^{11}$ First, the investigator (CJB) familiarised himself with the survey data, after which a thematic framework of themes and subthemes was created. The framework was indexed, and this was applied to the sections of text from the survey data. Data for each main theme were divided into relevant subthemes. Each table allowed for emerging concepts or suggestions to be identified, and was used to determine associated changes to the leaflet required. Accuracy of information and themes in tables were checked by a second investigator (MSR) before changes to the leaflet were made. In the presence of conflicting suggestions between different experts or compared to the current evidence base, choice of action was made following discussion between the two investigators. The Framework Analysis, and changes in response or concerns regarding changes, were provided to each of the participating experts, and further feedback was sought.

\section{Feedback from patients (phase 3 )}

We asked 20 patients diagnosed with PFP from 7 clinics or outpatient departments to provide feedback related to the clarity and adequacy of information contained in the final version of the leaflet (ie, following the second round of feedback from the experts), and to determine additional educational resource needs (see online supplementary file 2 ). To ensure wider external applicability of the leaflet and education resources developed in the future, we consulted patients of therapists from both private and public sectors in a range of English speaking countries (Australia, New Zealand, the USA, Canada and the UK). Age and duration of symptoms of patients with PFP surveyed ranged from 18 to 58 (average $=33$ ) years and 1 to $360 \quad$ (average $=55$ ) months, respectively. Of the 15 who chose to provide their professional backgrounds, 9 worked in jobs requiring a university degree (eg, lawyer), three worked in fields which did not necessitate tertiary education (eg, tradesman), and three were university students. Findings and suggestions from this process were clustered together and tabulated where possible.

\section{RESULTS}

The 'Framework Analysis' derived via expert consultation (phase 2) identified five themes (24 subthemes), including 'General format', 'General considerations', suggestions for changes to 'Why do I have knee pain?' and 'Management' sections, and 'Figures' (see online supplementary file 3). Changes in response or concerns regarding suggested changes resulting from expert consultation are outlined in the same tables (see online supplementary file 3). Key changes to the leaflet included the use of the term 'therapist' instead of 'physiotherapist' to make the leaflet more applicable to different health professions, stating that rest may be needed to settle symptoms before starting exercise rehabilitation, reducing emphasis on biomechanics and emphasising 'overuse' may be the key reason behind PFP development, emphasising the need for assessment and appropriate guidance from the 
KEY FACTORS AND

TREATMENT OPTIONS

(See inside for greater details)

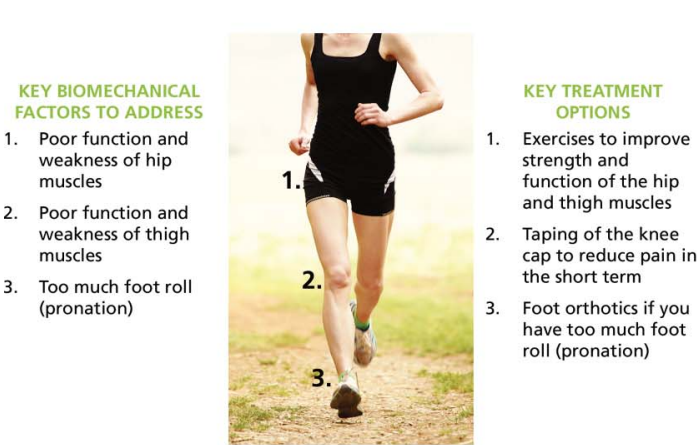

PRIORITIES TO MANAGE YOUR PATELLOFEMORAL PAIN

1. If you think you have patellofemoral pain, you should seek help as early as possible - this will improve your chances of a successful recovery.

There are many effective treatment options which you should discuss with your treating therapist.

Your recovery will be best if you actively participate in you rehabilitation

4. Appropriately modifying your physical activity level is often the first step towards successful recovery.

5 Ensure you slowly and safely build up your physical activity levels (Figure 5).

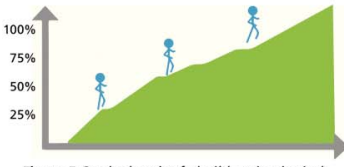
Figure 5 Gradual and safe build up in physical
activity levels

\section{WHAT MIGHT CAUSE MY KNEE PAIN?}

Excessive loading or varied and rapid increases to physical activity which your knee cannot cope with (Figure 1) are thought to contribute to pain development. Poor biomechanics (movement) can also contribute, with the knee cap thought to move toward the outside of the knee (Figure 2), stopping it from tracking normally in its groove. A number of factors can lead to this poor tracking (Figure 3). There are numerous other contributing factors to patellofemora pain including the structure of your disease, which you may wish to speak to your therapist about.

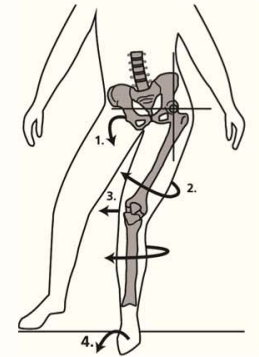

Figure 3 Movement factors contributing
to abnormal tracking of the knee cap.

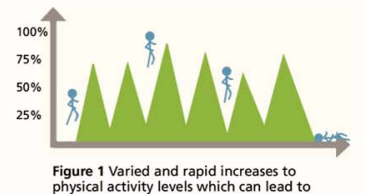

Figure 1 Varied and rapid increases to
physical activity levels which can lead to physical activity level
patellofemoral pain

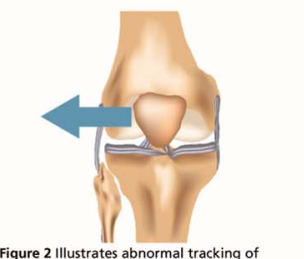

Figure 2 Illust

1. Pelvis drops on opposite side, placing increased tension on the outside of the leg and pulling th knee cap outward.

2. Hip collapses inward and rolls under the knee cap due to poor the muscles.

muscles are weak or

function poorly, meaning there is inadequate support for the kne and knee cap.

4. Foot rolls in too much, causing the shin and knee to collapse inward under the knee cap.

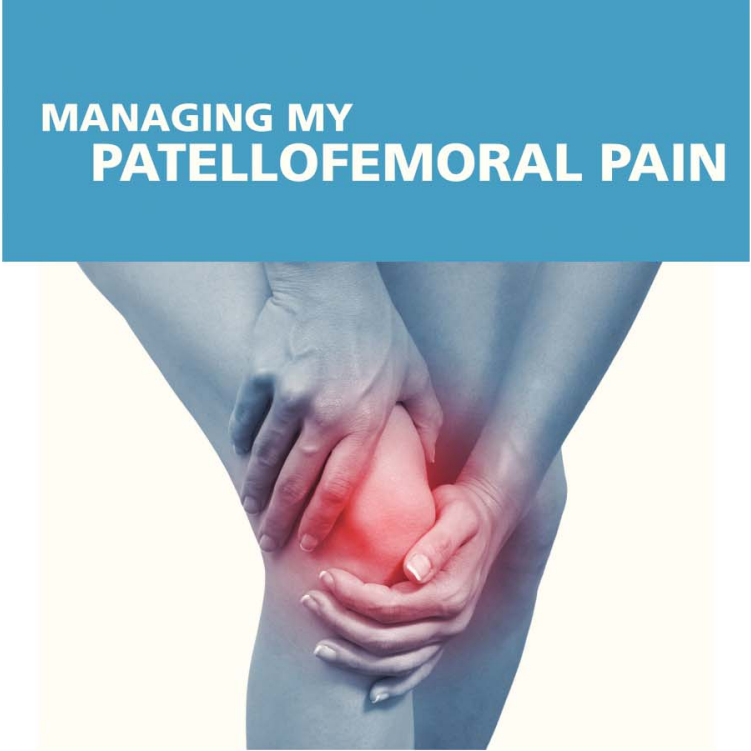

Patellofemoral pain (pain around, behind or under the knee cap) is very common, and affects both males and females of all activity levels. It often results in pain during simple daily activities such as walking, running, sitting, squatting and walking up and down stairs.

Patellofemoral pain has many causes, and as such there are a lot of reatment options available. The information contained within this your knee pain. It is ryou on the most appropriaspropiat your knee pain. It is recommended you see an appropriately qualified

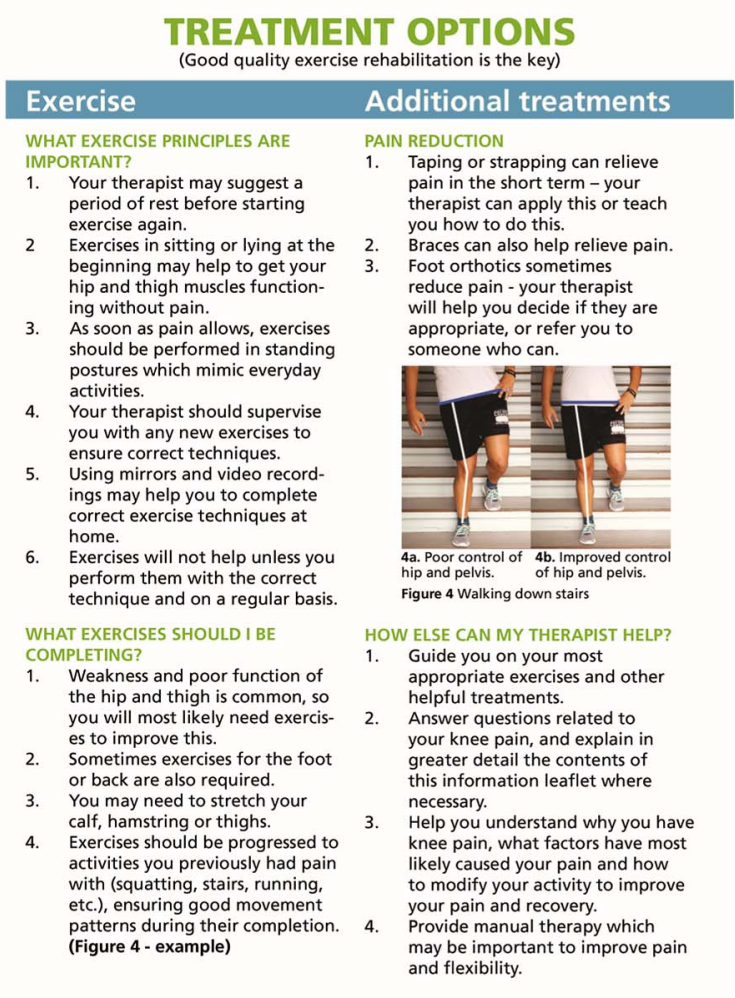

Figure 1 The 'Managing My Patellofemoral Pain' education leaflet.

patient's treating therapist, and removing direct reference to acupuncture as a possible pain reducing intervention, which is consistent with the final published guide. ${ }^{2}$ Further feedback following additional consultation with experts was provided by 11 of the 21 experts, and involved only typographical errors and sentence structure (see online supplementary file 4). No further changes to content or structure were suggested.

Feedback derived from patients (phase 3) was generally positive (see online supplementary file 5), and no 
further modifications were made to the leaflet. Patients also provided a number of suggestions for additional education resources (see online supplementary file 5). These included a dedicated website to facilitate patient education; further guidance and videos related to beneficial exercises; more detailed information on diagnosis, causation, management and prognosis; and a list of therapists who could be recommended to help.

The title of the leaflet is 'Managing My Patellofemoral Pain' and the main topics include 'What might cause my knee pain?', and 'Treatment options', which are divided into exercise and additional treatments. The resulting leaflet can be seen in figure 1 . This version of the leaflet is structured to print double-sided on A4 paper and be folded, allowing easy use in physical form as an adjunct to facilitate education with patients. Additionally, a freely available online version can be found here (http://bit. ly/11u1TtE). Readability of the text contained in the final version of the leaflet was calculated as 10.5 using the 'Fleish-Kincaid Grade Level' in Microsoft Word.

\section{DISCUSSION}

Appropriate patient education is essential to effective PFP management. ${ }^{2}$ The 'Managing My Patellofemoral Pain' education leaflet developed in this study provides a valuable resource for patients, clinicians and researchers to assist the provision of education and improve knowledge retention. The leaflet is designed as an adjunct to facilitate patient education when providing tailored multimodal intervention to individuals with $\mathrm{PFP}^{2}$ and should not be used as a stand-alone intervention. Patient education has proven effective in several long-standing musculoskeletal conditions, and it is therefore an essential part of multimodal treatment. ${ }^{3}{ }^{12}$ Importantly, however, knowledge retention from education interventions is usually low if information is only provided verbally. ${ }^{4}{ }^{5}$ Therefore, the patient education leaflet created may enhance knowledge retention and ultimately improve patient care for individuals with PFP. There are no specific rules/guides on how the leaflet should be used in clinical practice, and its implementation may depend on available time, and individual therapist and patient preferences. However, the therapist is recommended to go through the leaflet with their patient and allow time for, and encourage, additional questions not covered by the leaflet.

\section{Limitations}

Not all experts agreed on all content in the leaflet, but this is an inherent limitation in this form of qualitative research. For example, there were some suggestions from experts to reduce the leaflet's content, while other suggestions indicated that the leaflet's comprehensiveness was its strength. Considering that shortening the leaflet would require removal of information included in the 'Best Practice Guide to Patellofemoral Pain Management', maintaining the same level of content was determined to be the most appropriate action. This decision was validated with positive patient feedback during phase 3 , including praise for the leaflet's comprehensiveness (see online supplementary file 5).

Additional patient feedback indicated that the education leaflet does not answer all their questions. Instead of writing a more extensive and longer patient education leaflet, only the most relevant information based on the recently published 'Best Practice Guide to Conservative Management of Patellofemoral Pain ${ }^{2}$ was included in the final version. This choice was made since increasing the leaflet's length may cause some patients to lose interest and not read the entire content, or it may take too long to go through the entire leaflet in clinical practice where time is often limited. When using the leaflet, it is important for the therapist and patient to understand that it is intended to be an adjunct, and the therapist should possess the knowledge to answer additional questions and provide further relevant education.

The 'Managing My Patellofemoral Pain' education leaflet was designed for adult patients, and only feedback from adults with PFP was sought. Since PFP is very common among adolescents, ${ }^{13}$ future iterations of the leaflet should consider including an adolescents-specific version. Information for this specific population may need to be delivered in another format, for example, video or online.

\section{Future research}

Future studies should investigate the impact of the 'Managing My Patellofemoral Pain' education leaflet on health behaviour, knowledge retention and patient outcomes. Continued development of the leaflet is also needed as new knowledge accumulates. Furthermore, to improve its wider applicability, there is a need to translate it to non-English languages and integrate its contents into other media platforms including video, audio and web-based applications.

To address the need for more information and education resources, feedback from patients in this study suggested the need for a dedicated website to facilitate patient education. It was suggested that this should include further guidance and videos related to beneficial exercises; more detailed information on diagnosis, causation, management and prognosis; and a list of therapists who could be recommended to help. Further work is now needed to improve available resources to complement information provided in the 'Managing My Patellofemoral Pain' education leaflet.

\section{CONCLUSION}

The 'Managing My Patellofemoral Pain' education leaflet begins to address the absence of educational resources for PFP management, and provides a valuable resource for patients, clinicians and researchers to use alongside a multimodal approach to PFP management. Importantly, the leaflet may enhance the translation of the current evidence base into clinical practice. Investigating the leaflet's impact on health behaviour, 
knowledge retention and patient outcomes, alongside translation to non-English languages, is now needed. Furthermore, integration and expansion of the leaflet's contents into other media platforms including video, audio and web-based applications may enhance its capacity to improve patient care.

\section{Author affiliations}

${ }^{1}$ Complete Sports Care, Melbourne, Victoria, Australia

${ }^{2}$ La Trobe Sport and Exercise Medicine Research Centre, School of Allied

Health, La Trobe University, Melbourne, Victoria, Australia

${ }^{3}$ Pure Sports Medicine, London, UK

${ }^{4}$ Centre for Sports and Exercise Medicine, Queen Mary University of London, UK

${ }^{5}$ Department of Health Science and Technology, Faculty of Medicine, Center for Sensory-Motor Interaction (SMI), Aalborg University, Aalborg, Denmark ${ }^{6}$ Department of Occupational Therapy and Physiotherapy, Aalborg University Hospital, Aalborg, Denmark

${ }^{7}$ Research Unit for General Practice and Department of Clinical Medicine, Aalborg University, Denmark

\section{Twitter Follow Christian Barton at @DrChrisBarton}

Acknowledgements The authors would like to thank the 21 experts who gave their precious time and expertise; and the 20 patients with patellofemoral pain who provided further insight into the education leaflet's value. Additionally, thanks to Esther Williams for help with designing the layout and producing the preliminary and final versions of the education leaflet.

Contributors CJB and MSR conceived and planned the study. CJB collected the survey responses. CJB and MSR analysed and interpreted all data and completed the write-up of the manuscript.

Competing interests None declared.

Provenance and peer review Not commissioned; externally peer reviewed.

Open Access This is an Open Access article distributed in accordance with the Creative Commons Attribution Non Commercial (CC BY-NC 4.0) license, which permits others to distribute, remix, adapt, build upon this work noncommercially, and license their derivative works on different terms, provided the original work is properly cited and the use is non-commercial. See: http:// creativecommons.org/licenses/by-nc/4.0/

\section{REFERENCES}

1. Taunton JE, Ryan MB, Clement DB, et al. A retrospective case-control analysis of 2002 running injuries. Br J Sports Med 2002;36:95-101.

2. Barton CJ, Lack S, Hemmings S, et al. The 'Best Practice Guide to Conservative Management of Patellofemoral Pain': incorporating level 1 evidence with expert clinical reasoning. $\mathrm{Br} J$ Sports Med 2015;49:923-34.

3. Fernandes L, Hagen KB, Bijlsma JW, et al. EULAR recommendations for the non-pharmacological core management of hip and knee osteoarthritis. Ann Rheum Dis 2013;72:1125-35.

4. Oshodi TO. The impact of preoperative education on postoperative pain. Part 1. Br J Nurs 2007;16:706-10.

5. Johnson A, Sandford J. Written and verbal information versus verbal information only for patients being discharged from acute hospital settings to home: systematic review. Health Educ Res 2005;20:423-9.

6. Rathleff MS, Roos EM, Olesen JL, et al. Exercise during school hours when added to patient education improves outcome for 2 years in adolescent patellofemoral pain: a cluster randomised trial. Br J Sports Med 2015;49:406-12.

7. Collins N, Crossley K, Beller E, et al. Foot orthoses and physiotherapy in the treatment of patellofemoral pain syndrome: randomised clinical trial. BMJ 2008;337:a1735.

8. Clark DI, Downing N, Mitchell J, et al. Physiotherapy for anterior knee pain: a randomised controlled trial. Ann Rheum Dis 2000;59:700-4.

9. van Linschoten R, van Middelkoop M, Berger MY, et al. Supervised exercise therapy versus usual care for patellofemoral pain syndrome: an open label randomised controlled trial. BMJ 2009;339:b4074.

10. Witvrouw E, Callaghan MJ, Stefanik JJ, et al. Patellofemoral pain: consensus statement from the 3rd International Patellofemoral Pain Research Retreat held in Vancouver, September 2013. Br J Sports Med 2014;48:411-14.

11. Mays N, Pope C. Qualitative research in healthcare: analysing qualitative data. 2nd edn. BMJ Books, 1999.

12. Riemsma RP, Kirwan JR, Taal E, et al. Patient education for adults with rheumatoid arthritis. Cochrane Database Syst Rev 2003;(2): CD003688.

13. Rathleff MS, Roos EM, Olesen JL, et al. Lower mechanical pressure pain thresholds in female adolescents with patellofemoral pain syndrome. J Orthop Sports Phys Ther 2013;43:414-21. 